\title{
A NOTE ON COCYCLES IN VON NEUMANN ALGEBRAS
}

\author{
KICHI-SUKE SAITO
}

ABSTRACT. In this note, we study the comparison theory for cocycles in von Neumann algebras. In particular, we investigate quasi-equivalent cocylces.

In [1], Connes and Takesaki studied a comparison theory for cocycles with respect to a given continuous group action on a von Neumann algebra. This theory will give rise, via the Connes cocycle theorem $[1,3.1,3.5]$, to a corresponding comparison theory for weights on von Neumann algebras. Further, Muhly and the author in [2] proved that when a von Neumann algebra $M$ is in stardard form, there is essentially a one-to-one correspondence between invariant subspaces of an analytic subalgebra of $M$ which is determined by an action $\left\{\alpha_{t}\right\}_{t \in \mathbf{R}}$ and cocylces for $\left\{\alpha_{t}\right\}_{t \in \mathbf{R}}$.

In this note, we shall develop a comparison theory for cocycles in a von Neumann algebra, in particular, a finite von Neumann algebra, and apply it to a comparison theory for invariant subspaces in von Neumann algebras.

Let $M$ be a von Neumann algebra and let $G$ be a locally compact group. Let $\alpha: G \rightarrow \operatorname{Aut}(M)$ be a continuous action of $G$ on $M$. As in $[4,20.1]$, recall that a cocycle is an $s^{*}$-continuous function $a: G \ni s \rightarrow a(s) \in M$ with the properties:

$$
a(s t)=a(s) \alpha_{s}(a(t)) \quad \text { and } \quad a\left(s^{-1}\right)=\alpha_{s}^{-1}\left(a(s)^{*}\right), \quad s, t \in G,
$$

and that the set of all cocycles is denoted by $Z_{\alpha}(G, M)$. If $a \in Z_{\alpha}(G, M)$, then the elements $a(s) \in M$ are partial isometries:

$$
a(s) a(s)^{*}=a(1), \quad a(s)^{*} a(s)=\alpha_{s}(a(1)), \quad s \in G ;
$$

in particular, $a(1)$ is a projection, where 1 means the identity of $G$. For each $s \in G$, we set

$$
{ }_{a} \alpha_{s}(x)=a(s) \alpha_{s}(x) a(s)^{*}, \quad x \in M_{a(1)}, s \in G .
$$

Then ${ }_{a} \alpha: G \rightarrow \operatorname{Aut}\left(M_{a(1)}\right)$ is a continuous action whose centralizer is denoted by $M^{a}=\left(M_{a(1)}\right)^{a^{\alpha}}$. If $p \in \operatorname{Proj}\left(M^{a}\right)$, then the map $s \in G \rightarrow p a(s) \in M$ is a cocycle in $M$. We call it a subcocycle of $a$ and denote it by $a^{p}$.

Let $F_{2}$ be the type $I_{2}$-factor with the system of matrix units $\left\{e_{i j}\right\}_{1 \leq i, j \leq 2}$. We shall identify $M \otimes F_{2}$ with $\operatorname{Mat}_{2}(M)$ in the usual way. Let $\iota: G \rightarrow \operatorname{Aut}\left(F_{2}\right)$ be the trivial action. Then $\alpha \otimes \iota: G \rightarrow \operatorname{Aut}\left(M \otimes F_{2}\right)$ is a continuous action. Given $a, b \in Z_{\alpha}(G, M)$, we define the balanced cocycle $c=c(a, b)$ associated with the cocycles $a$ and $b$ by

$$
c(s)=a(s) \otimes e_{11}+b(s) \otimes e_{22}=\left(\begin{array}{cc}
a(s) & 0 \\
0 & b(s)
\end{array}\right), \quad s \in G,
$$

Received by the editors December 17, 1985 and, in revised form, April 14, 1986.

1980 Mathematics Subject Classification (1985 Revision). Primary 46L10; Secondary 47A15. 
and the set $I(a, b)$ by

$$
I(a, b)=\left\{x \in a(1) M b(1): x b(s)=a(s) \alpha_{s}(x) \text { for all } s \in G\right\} .
$$

DEFINITION 1. We say that $a, b \in Z_{\alpha}(G, M)$ are equivalent and write $a \simeq b$ if there exists an element $c \in M$ such that

$$
a(s)=c^{*} b(s) \alpha_{s}(c), \quad b(s)=c a(s) \alpha_{s}\left(c^{*}\right), \quad s \in G .
$$

We write $a \lesssim b$ if $a \simeq b^{p}$ for some $p \in \operatorname{Proj}\left(M^{b}\right)$. Further, we say that $a$ and $b$ are disjoint and write $a_{0}^{\dagger} b$ if $a(1) \otimes e_{11}$ and $b(1) \otimes e_{22}$ are centrally orthogonal in $M_{2}^{c}$. We say also that $a$ and $b$ are quasi-equivalent and write $a \sim b$ if $a(1) \otimes e_{11}$ and $b(1) \otimes e_{22}$ have the same central support in $M_{2}^{c}$. We write $a \precsim k$ if $a \sim b^{p}$ for some $p \in \operatorname{Proj}\left(M^{b}\right)$.

Let $a, b \in Z_{\alpha}(G, M)$. If $a_{\circ}^{\mid} b$, then $I(a, b)=\{0\}$ (cf. [4, Proposition 20.2]). We now study the structure of $I(a, b)$ when $a$ and $b$ are not disjoint. Assume that $I(a, b) \neq\{0\}$. Let $x \in I(a, b), x \neq 0$. If $x=v|x|$ is the polar decomposition of $x$, then $|x| \in I(b, b)=M^{b}$ and $v \in I(a, b)$. Thus we have $v^{*} v \leq b(1)$ and $v v^{*} \leq a(1)$. We put

$$
p(a, b)=\sup \left\{v^{*} v: v \text { is a partial isometry in } I(a, b)\right\}
$$

and

$$
q(a, b)=\sup \left\{v v^{*}: v \text { is a partial isometry in } I(a, b)\right\},
$$

respectively. Hence it is clear that the $\sigma$-weakly closed linear span $J$ of $I(a, b)^{*} I(a, b)$ is a $\sigma$-weakly closed 2-sided ideal of $M^{b}$. Thus there exists a central project $e_{0}$ in $M^{b}$ such that $J=M^{b} e_{0}$. By the definition of $p(a, b)$, we easily have $p(a, b)=e_{0}$ and so $p(a, b)$ is a central projection in $M^{b}$. Furthermore, $p(a, b)$ is the least central projection in $M^{b}$ such that $x p(a, b)=x(x \in I(a, b))$. Similarly $q(a, b)$ is the least central projection in $M^{a}$ such that $q(a, b) x=x$ for ali $x \in I(a, b)^{*}=I(b, a)$. For simplicity, we put $p(a, b)=p$ and $q(a, b)=q$, respectively.

Proposition 2. Let $a, b \in Z_{\alpha}(G, M)$. Then $\left.a^{a(1)-q}\right|_{\circ} b$ and $a^{q} \sim b^{p}$.

ProOF. Put $r=a(1)-q$. Then it is sufficient to prove that $I\left(a^{r}, b\right)=\{0\}$. Since $r \in M^{a}, a(t) \alpha_{t}(r) a(t)^{*}=r$. Let $x \in I\left(a^{r}, b\right)$. Then

$$
\begin{aligned}
x b(t) & =r a(t) \alpha_{t}(x)=a(t) \alpha_{t}(r) a(t)^{*} a(t) \alpha_{t}(x) \\
& =a(t) \alpha_{t}(r) \alpha_{t}(a(1)) \alpha_{t}(x)=a(t) \alpha_{t}(r x)=a(t) \alpha_{t}(x) .
\end{aligned}
$$

Thus $I\left(a^{r}, b\right) \subset I(a, b) \cap r M b(1)=\{0\}$. This implies that $a^{r !} b$.

Next we shall prove that $a^{q} \sim b^{p}$. To prove this, it is sufficient to prove that $q \otimes e_{11}$ and $p \otimes e_{22}$ have the same central support in $M_{2}^{c}$. Let $\left(\begin{array}{ll}x & y \\ z & w\end{array}\right)$ be the central support of $q \otimes e_{11}=\left(\begin{array}{ll}q & 0 \\ 0 & 0\end{array}\right)$ in $M_{2}^{c}$. By [4, Proposition 20.2], it is clear that $x$ (resp. $y$ ) is a central projection in $M^{a}\left(\right.$ resp. $\left.M^{b}\right), y=z=0$, and $x d=d w$ for all $d \in I(a, b)$. On the other hand,

$$
\left(\begin{array}{ll}
q & 0 \\
0 & 0
\end{array}\right) \leq\left(\begin{array}{cc}
x & 0 \\
0 & w
\end{array}\right) \leq\left(\begin{array}{ll}
q & 0 \\
0 & p
\end{array}\right)
$$

and so $q=x$ and $w \leq p$. Since $d=q d=d w$ for all $d \in I(a, b)$ and $p$ is the least central projection in $M^{b}$ such that $d p=d$ for all $d \in I(a, b), p=w$. Thus the central support of $q \otimes e_{11}$ is $q \otimes e_{11}+p \otimes e_{22}$. Similarly, we have the central support of $p \otimes e_{22}=q \otimes e_{11}+p \otimes e_{22}$. Thus $a^{q} \sim b^{p}$. This completes the proof. 
If $M$ is $\sigma$-finite and $a, b \in Z_{\alpha}(G, M)$ are of infinite multiplicity, then $a \simeq b$ is equivalent to $a \sim b[4,20.2]$. However, if $a, b \in Z_{\alpha}(G, M)$ are not necessarily of infinite multiplicity, then we have the following theorem.

THEOREM 3. Let $a, b \in Z_{\alpha}(G, M)$. If $a \precsim b$, then there is a family $\left\{v_{\gamma}\right\}_{\gamma \in \Gamma}$ of partial isometries in $I(a, b)$ with the following properties:

(1) $v_{\gamma}^{*} v_{\gamma}=0$ if $\gamma \neq \lambda$;

(2) $\sum_{\gamma \in \Gamma} v_{\gamma} v_{\gamma}^{*}=q$;

(3) $I(a, b)=\sum_{\gamma \in \Gamma} v_{\gamma} M^{b}$,

i.e. each $x \in I(a, b)$ can be written as $\sum_{\gamma \in \Gamma} v_{\gamma} x_{\gamma}$ for some $x_{\gamma} \in M^{b}$, where the sum converges in the $\sigma$-weak operator topology. In this case, we have $a(t)=$ $\sum_{\gamma \in \Gamma} v_{\gamma} b(t) \alpha_{t}\left(v_{\gamma}^{*}\right)$.

Proof. By Zorn's lemma, there exists a maximal family $\left\{v_{\gamma}\right\}_{\gamma \in \Gamma}$ of partial isometries in $I(a, b)$ such that $v_{\gamma}^{*} v_{\lambda}=0(\gamma \neq \lambda)$. Then we can prove that $\sum_{\gamma \in \Gamma} v_{\gamma} v_{\gamma}^{*}=q$. Assume that $q_{0}=q-\sum_{\gamma \in \Gamma} v_{\gamma} v_{\gamma}^{*} \neq 0$. Then, by the definition of $q$, there exists a partial isometry $v$ in $I(a, b)$ such that $v v^{*} q_{0} \neq 0$. By the Comparability Theorem, there are a central projection $z$ in $M^{a}$ and partial isometries $u_{1}, u_{2}$ in $M^{a}$ such that $u_{1}^{*} u_{1}=z q_{0}, u_{1} u_{1}^{*} \leq z v v^{*}, u_{2}^{*} u_{2}=(q-z) v v^{*}$, and $u_{2} u_{2}^{*} \leq(q-z) q_{0}$. Then we have either $u_{1} \neq 0$ or $u_{2} \neq 0$. If $u_{1} \neq 0$, then we set $v_{1}=u_{1}^{*} z v$. Hence

$$
v_{1} v_{1}^{*}=u_{1}^{*} z v v^{*} z u_{1}=u_{1}^{*} u_{1} u_{1}^{*} u_{1}=u_{1}^{*} u_{1}=z q_{0} \leq q_{0} .
$$

Similarly, if $u_{2} \neq 0$, then we put $v_{2}=u_{2}(q-z) v$. In both cases, we have a contradiction. Thus $q=\sum_{\gamma \in \Gamma} v_{\gamma} v_{\gamma}^{*}$. Further, for each $x \in I(a, b), x=q x=$ $\sum_{\gamma \in \Gamma} v_{\gamma} v_{\gamma}^{*} x$. Since $v_{\gamma}^{*} x \in I(a, b)^{*} I(a, b) \subset I(b, b)=M^{b}$, put $x_{\gamma}=v_{\gamma}^{*} x$. Then $x=\sum_{\gamma \in \Gamma} v_{\gamma} x_{\gamma}$ and so $I(a, b)=\sum_{\gamma \in \Gamma} v_{\gamma} M^{b}$. Finally, we have

$$
\begin{aligned}
\sum_{\gamma \in \Gamma} v_{\gamma} b(t) \alpha_{t}\left(v_{\gamma}^{*}\right) & =\sum_{\gamma \in \Gamma} a(t) \alpha_{t}\left(v_{\gamma}\right) \alpha_{t}\left(v_{\gamma}^{*}\right)=\sum_{\gamma \in \Gamma} a(t) \alpha_{t}\left(v_{\gamma} v_{\gamma}^{*}\right) \\
& =a(t) \alpha_{t}\left(\sum_{\gamma \in \Gamma} v_{\gamma} v_{\gamma}^{*}\right)=a(t) \alpha_{t}(a(1))=a(t) a(t)^{*} a(t)=a(t) .
\end{aligned}
$$

This completes the proof.

Next we study the special case of Theorem 3 .

THEOREM 4. Let $M$ be a finite von Neumann algebra and $a, b \in Z_{\alpha}(G, M)$. Suppose that the center $\mathfrak{Z}\left(M^{b}\right)$ of $M^{b}$ is contained in the center $\mathfrak{Z}(M)$ of $M$. If $a \sim b$, then $a \simeq b$.

PROOF. Consider a maximal family $\left\{u_{\gamma}\right\}_{\gamma \in \Gamma}$ of partial isometries of $I(a, b)$ such that $u_{\gamma} u_{\gamma}^{*}$ are mutually orthogonal and $u_{\gamma}^{*} u_{\gamma}$ are mutually orthogonal. Put $v=\sum_{\gamma \in \Gamma} u_{\gamma}$. Then $v$ is a partial isometry of $I(a, b)$. Since $q=a(1)$, suppose that $q-v v^{*} \neq 0$. From the definition of $q$, as in the proof of Theorem 3, there exists a partial isometry $v_{1}$ in $I(a, b)$ such that $v_{1} v_{1}^{*} \leq q-v v^{*}$. Since $M$ is a finite von Neumann algebra, it is clear that $p=q=b(1)$. Let $T$ (resp. $T_{0}$ ) be the center valued trace of $M$ (resp. $\left.M^{b}\right)$. Since $\mathfrak{Z}\left(M^{b}\right) \subset \mathfrak{Z}(M)$, the restriction of $T$ to $M^{b}$ equals $T_{0}$. Hence we have

$$
\begin{aligned}
T_{0}\left(q-v^{*} v\right) & =T\left(q-v^{*} v\right)=T\left(q-v v^{*}\right) \geq T\left(v_{1} v_{1}^{*}\right) \\
& =T\left(v_{1} v_{1}^{*}\right)=T_{0}\left(v_{1}^{*} v_{1}\right)
\end{aligned}
$$


By [5, p. 314, Corollary 2.8], $v_{1}^{*} v_{1} \precsim q-v^{*} v$ in $M^{b}$. Thus there is a partial isometry $u$ in $M^{b}$ such that $u^{*} u=v_{1}^{*} v_{1}$ and $u u^{*} \leq q-v^{*} v$. Put $v_{2}=v_{1} u^{*}$. Then $v_{2}^{*} v_{2}=u v_{1}^{*} v_{1} u^{*}=u u^{*} u u^{*} \leq q-v^{*} v$ and $v_{2}^{*} v_{2}=v_{1} u^{*} u v_{1}^{*}=v_{1} v_{1}^{*} \leq q-v v^{*}$. Since $v_{2}$ is a nonzero partial isometry in $I(a, b)$, we have a contradiction. Thus $v v^{*}=v^{*} v=q$. Then $I(a, b)=v M^{b}$ and so $a \simeq b$. This completes the proof.

COROLlaRY 5. Let $M$ be a finite von Neumann algebra and $a \in Z_{\alpha}(G, M)$. If $M^{\alpha}$ is a factor, then $a_{\circ}^{\mid} 1$ or $a \precsim 1$. Further, if $a$ is a unitary cocycle of $M$, then $a_{\circ}^{\dagger} 1$ or $a \simeq 1$.

Finally we consider the form of invariant subspaces. We refer the reader to [2] for the definitions and notations about invariant subspaces. Let $M$ be a von Neumann algebra acting on the noncommutative Lebesgue space $L^{2}(M)$ in the sense of Haagerup (see [6]). Let $\left\{\alpha_{t}\right\}_{t \in \mathbf{R}}$ be a $\sigma$-weakly continuous, one-parameter group of *automorphisms of $M$. Then there is a uniquely unitary group $\left\{U_{t}\right\}_{t \in \mathbf{R}}$ on $L^{2}(M)$ such that $R_{\alpha_{t}(x)}=U_{t} R_{x} U_{t}^{*}$ for all $x \in M$ and $t \in \mathbf{R}$. In this note, we consider the version of right-invariant subspaces. By [2, Theorem 3.1], we have the following theorem.

THEOREM 5. Let $\mathfrak{M}$ be a right-pure, right-invariant subspace of $L^{2}(M)$ that is left-normalized (resp. right-normalized). Then there are a projection $p$ in $M, a$ strongly continuous unitary group $\left\{V_{t}\right\}_{t \in \mathbf{R}}$ of $\mathbf{R}$ on $L_{p} L^{2}(M)$, and $a \in Z_{\alpha}(G, M)$ such that

(1) $R_{\alpha_{t}(x)} L_{p}=V_{t} R_{x} V_{t}^{*}$ for all $x \in M, t \in \mathbf{R}$;

(2) $V_{t}=L_{a(t)} U_{t}$ for all $t \in \mathbf{R}$;

(3) $\mathfrak{M}=F[0, \infty) L_{p} L^{2}(M)$ (resp. $\left.\mathfrak{M}=F(0, \infty) L_{p} L^{2}(M)\right)$, where $F$ is the spectral measure for $V$ on $L_{p} L^{2}(M)$.

Let $\mathfrak{M}$ be a left-normalized, right-pure, right-invariant subspace of $L^{2}(M)$ with $a \in Z_{\alpha}(\mathbf{R}, M)$. If $a \precsim 1$, by Theorem 3 , then there exists a family $\left\{v_{\gamma}\right\}_{\gamma \in \Gamma}$ of partial isometries in $I(a, 1)$ such that $a(t)=\sum_{\gamma \in \Gamma} v_{\gamma} \alpha_{t}\left(v_{\gamma}^{*}\right)$. Then we have

$$
\sum_{\gamma \in \Gamma} L_{v_{\gamma}} U_{t} L_{v_{\gamma}^{*}}=\sum_{\gamma \in \Gamma} L_{v_{\gamma}} L_{\alpha_{t}\left(v_{\gamma}^{*}\right)} U_{t}=L_{a(t)} U_{t}=V_{t} .
$$

By the uniqueness of spectral decomposition, we have

$$
F[\lambda, \infty)=\sum_{\gamma \in \Gamma} L_{v_{\gamma}} P[\lambda, \infty) L_{v_{\gamma}^{*}}
$$

in particular, $F[0, \infty)=\sum_{\gamma \in \Gamma} L_{v_{\gamma}} P[0, \infty) L_{v_{\gamma}^{*}}$, where $P$ is the spectral measure of $\left\{U_{t}\right\}_{t \in \mathbf{R}}$. Hence

$$
\begin{aligned}
\mathfrak{M} & =F[0, \infty) L^{2}(M)=\sum_{\gamma \in \Gamma} L_{v_{\gamma}} P[0, \infty) L_{v_{\gamma}^{*}} L^{2}(M) \\
& =\sum_{\gamma \in \Gamma} L_{v_{\gamma}} P[0, \infty) L_{v_{\gamma}^{*}} L_{v_{\gamma}} L^{2}(M)=\sum_{\gamma \in \Gamma} L_{v_{\gamma}} L_{v_{\gamma}^{*}} L_{v_{\gamma}} P[0, \infty) L^{2}(M) \\
& =\sum_{\gamma \in \Gamma} L_{v_{\gamma}} P[0, \infty) L^{2}(M)=\sum_{\gamma \in \Gamma} \oplus L_{v_{\gamma}} \mathbf{H}^{2},
\end{aligned}
$$

because $v_{\gamma}^{*} v_{\gamma} \in M^{\alpha}$ and $P[0, \infty) \in L\left(M^{\alpha}\right)^{\prime}$. Thus we have the following proposition. 
PROPOSITION 6. Let $\mathfrak{M}$ be a left-normalized (resp. right-normalized), rightpure, right-invariant subspace of $L^{2}(M)$ with $a \in Z_{\alpha}(\mathbf{R}, M)$. If $a \precsim 1$, then there exists a family $\left\{v_{\gamma}\right\}_{\gamma \in \gamma}$ of partial isometries of $I(a, 1)$ such that $\mathfrak{M}=\sum_{\gamma \in \Gamma} \oplus L_{v_{\gamma}} \mathbf{H}^{2}$ (resp. $\left.\sum_{\gamma \in \Gamma} \oplus L_{v_{\gamma}} \mathbf{H}_{0}^{2}\right)$.

\section{REFERENCES}

1. A. Connes and M. Takesaki, The flow of weights on factors of type III, Tôhoku Math. J. 29 (1977), 473-575.

2. P. S. Muhly and K.-S. Saito, Analytic subalgebras of von Neumann algebras, Canad. J. Math. (to appear).

3. K.-S. Saito, Nonselfadjonit subalgebras associated with compact abelian group actions on finite von Neumann algebras, Tôhoku Math. J. 34 (1982), 485-494.

4. S. Stratila, Modular theory in operator algebras, Abacus Press, Tunbridge, England, 1981.

5. M. Takesaki, Theory of operator algebras. I, Springer-Verlag, Berlin and New York, 1979.

6. M. Terp, $L^{p}$-spaces associated with von Neumann algebras, Rapport No. 3 , The University of Odense, 1981.

Department of Mathematics, Faculty of Science, Nigata University, NIIGATA 950-21, JAPAN 